\title{
Selective decontamination of the gastrointestinal tract: a possible strategy to reduce infection in high- risk populations
}

\begin{abstract}
The diverse microbial environment in the digestive tract, influences the human host's immune response and subsequent development of infection. In patients at high risk of infection, strategies to help reduce risk are key. Selective decontamination of the gastrointestinal tract has emerged as one of these potential strategies, with the two common forms being selective oral decontamination (SOD) or selective digestive decontamination (SDD).

SOD and SDD can take place using a variety of different, non-absorbable antibiotic regimens. Effective regimens have been reported with particular decrease in numbers of pathogenic gram-negative bacteria and resulting decrease in morbidity and mortality associated with bacteraemia. Work in this area has predominantly been in the area of critical care but there is increasing focus on using this intervention to reduce infection rates and anastamotic leaks in elective gastrointestinal surgical procedures.

The cost of such interventions as well as possible increase in antimicrobial resistance have been suggested as possible stumbling blocks to their uptake in routine clinical care. In this review, the role of SDD and SOD, including indications and limitations, will be examined to offer advice on their potential use for the practising clinician.
\end{abstract}

Keywords: Selective decontamination, Digestive tract, Infection risk, Prophylaxis
Volume 5 Issue 6 - 2016

Nurulamin M Noor

Department of Medicine, University of Cambridge, United Kingdom

Correspondence: Nurulamin M Noor, Department of Medicine, University of Cambridge, Addenbrooke's Hospital, Cambridge, CB2 0QQ, United Kingdom, Tel +44I223 245I5I, Emailnn28I@cam.ac.uk

Received: August 26, 2016 | Published: December 16, 2016
Abbreviations: SOD, Selective Oral Decontamination; SDD, Selective Digestive Decontamination; ICU, Intensive Care Unit; MRSA, Methicillin Resistant Staphylococcus Aureus

\section{Introduction}

The microbial ecology in the human digestive tract is diverse and here environmental triggers, immune signals and genetic susceptibility can affect the human host's response to infection. ${ }^{1}$ Selective decontamination has emerged as a potential treatment for patients at high risk of infection such as those; in the critical care environments, undergoing invasive surgical operations, or with significant immunosuppression. Selective decontamination aims to vastly reduce numbers of bacteria in the gastrointestinal (GI) tract and subsequently reduce the risk of infection. There are two main approaches: selective oropharyngeal decontamination (SOD) and selective digestive decontamination (SDD). ${ }^{2}$

SOD involves application of topical antibiotic paste to the orophyarynx and does not usually involve enteral or intravenous adjuvant antibiotics. ${ }^{2}$ This antibiotic paste is usually applied four times per day. SDD, on the other hand, consists of oropharyngeal and gastric application of non-absorbable antibiotics in addition to a short course of intravenous antibiotics. There are several different antibiotic regimens in use for selective decontamination and typically oropharyngeal and gastric antibiotics include either a combination or single use of: polymyxin E, tobramycin, and amphotericin $\mathrm{B} .^{3}$ These drugs are particularly active against potentially pathogenic gram-negative rods, Staphylococcus species, yeasts and fungi, but are relatively more sparing of less pathogenic organisms. ${ }^{3}$
There has been some debate over whether decreased bacterial carriage translates to reduced infections, ${ }^{4}$ whether this technique may lead to increased antibiotic resistance rates, ${ }^{5}$ and also whether this is a cost-effective strategy to reduce risk of infection. This review will aim to collate recent research on the use of selective decontamination using either SDD or SOD, and offer guidance on their potential use, including indications and limitations, for the practising clinician.

\section{Discussion}

Initially selective decontamination of GI tract was reported in 1980 in the setting of haemato-oncology. ${ }^{6}$ Subsequent applications of this management strategy were met with mixed response and use of different antibiotic regimens. ${ }^{7,8}$ Selective decontamination was applied to an intensive care setting, with a population of mixed pathologies with seemingly greater promise. ${ }^{9}$ SDD was performed using a triple antibiotic regimen of polymyxin E, tobramycin and amphotericin B. Over a 16 month period there was a striking reduction in colonisation of the digestive tract, particularly with gram-negative bacilli and there was a reduction in rates of infection versus control $(24 \%$ vs. $10 \%)$. Further work suggested that this technique resulted in a reduction in morbidity and mortality for intensive care patients and was achieved at a relatively low cost. ${ }^{10}$ Similar findings were found when patients were given a short 3 day course of intravenous antibiotics whilst starting on SDD for both medical and surgical patients on an intensive care unit. ${ }^{11}$

At this stage, stage there was a lack of randomised, double-blinded trial results in this field. Therein followed a period where attention on strategies to reduce infection risk, were focused elsewhere. In the intervening period, promising work was performed in mice, 
with different groups given five different regimens to assess microorganism translocation. Different antibiotic combinations were found to be effective at reducing growth of gram positive and gram-negative bacteria, but also for decreasing translocation of fungi, however the mechanism for this was not explored. Combinations which were found to be effective include; neomycin, erythromycin and metronidazole; polymyxin B and amikacin; polymyxin B, amikacin and amphotericin B. ${ }^{12}$

At the turn of the century, increasing focus began to be applied to the area of selective decontamination. A large randomised controlled trial was performed in 934 patients and showed that in a setting of a low antibiotic resistance intensive care unit, SDD decreased mortality $(23 \%$ vs. $15 \%, \mathrm{p}=0.002)$ and that there was also reduced colonisation with resistant gram-negative bacteria. ${ }^{13}$

At this stage, there was increasing application of SOD, which could perhaps achieve decontamination from oropharynx and in particular help decrease subsequent respiratory tract infections. Vancomycin use in SOD was associated with reduction in methicillinresistant staphylococcus aureus (MRSA) pneumonia in the Intensive Care Unit (ICU) setting. ${ }^{14}$ Both interventions seemed to reduce infections from gram-negative organisms, SOD wasassociated with a $33 \%$ and SDD was associated with a $45 \%$ reduction in the occurrence of intensive care unit-acquired gram-negative bacteraemia. ${ }^{15}$ Comparison has taken place between SDD and SOD as methods of selective decontamination. Initial findings looking particularly at respiratory tract bacteraemia in ICU patients and found that this was reduced using both SDD and SOD methods. ${ }^{16}$ A more recent network meta-analysis has shown a favourable effect on mortality from use of SDD in ICUs but that the effect of SOD is less certain. However this study did report that use of SOD was more effective than topical chlorhexidine in reducing risk of infection, a widely practised hospital intervention. $^{2}$

One key criticism of these interventional approaches is that they may lead to increased antimicrobial resistance, potentially selecting increasingly drug-resistant pathogenic strains. Indeed, an initial study suggested that rates of MRSA and ciprofloxacin resistance were higher in SDD groups rather than control groups. ${ }^{5}$ However this only followed up patients over a one year period, had low numbers of patients recruited and was a single centre, non-blinded study. Larger studies and over a longer period of time have subsequently found no effect on antimicrobial resistance at two years, ${ }^{17}$ and a cluster randomised trial of patients at 16 different centres also found no change to antibiotic resistance rates from patients receiving SDD or SOD. ${ }^{18}$ When comparing SOD and SDD antibiotic resistance, there were no differences in mortality detected, SDD seems to demonstrate lower rectal carriage of antibiotic-resistant gram-negative bacteria and subsequent bacteraemia, however SDD was associated with greater aminoglycoside-resistant gram-negative bacteria. ${ }^{19}$

There are limitations to the application of SDD or SOD. Of note, many studies have been single centre studies performed in intensive care units. There is the complex and varying nature of intensive care admissions, varying practices between countries and local antimicrobial resistance patterns showing great variety even from neighbouring regions. In addition, control groups of patients in ICU settings will frequently have varying prolonged courses of intravenous antibiotics, which will to varying degrees cause reduction in gut microbiota levels and alteration in composition. ${ }^{20}$ As a result, it may be difficult to apply findings to this heterogeneous group of patients.
Another potential stumbling block to using SDD or SOD in clinical practice is the possible expense of additional antibiotic courses. However, when routine use of selective decontamination examined, it was found to be less expensive and economically more efficient than placebo in reducing numbers of patients with infectious complications. ${ }^{21}$

Furthermore, the use of selective decontamination in patients undergoing high risk GI surgery has been examined and post-operative infection rates and anastamotic leaks seemed to be reduced with perioperative SDD. ${ }^{3}$ SOD has not been shown to be as effective in this cohort of patients. ${ }^{22}$ To date, there have no trials of pre-operative SDD or SOD prior to elective GI surgical operations. ${ }^{3}$ This could be an area for further exploration. The use of SDD was found to be cost effective at reducing infection in patients undergoing elective GI surgery. ${ }^{23}$ Trial recruitment is currently underway to further examine effect on morbidity and mortality following colorectal surgery. ${ }^{4}$

Perhaps beyond the scope of this review, but there have been promising applications of selective decontamination to reduce infection in; paediatrics, ${ }^{25,26}$ trauma, ${ }^{27,28}$ burns,${ }^{29}$ and following stroke. ${ }^{30}$

\section{Conclusion}

The microbial composition and environment in the gastrointestinal tract is closely linked to response to infection. Selective decontamination of the gastrointestinal tract has emerged as a possible therapeutic strategy to reduce risk of infection, in high-risk patient groups. There have been promising results from randomised trials in this area and in the short to medium term antibiotic resistance does not seem to be significantly increased. The most studied cohort of patients are those in a critical care setting. Given the heterogeneity of patients in ICUs, varying clinical practice, and antimicrobial resistance patterns, it may be difficult to apply findings to individual centres. Moreover, there is the significant confounder that patients in these environments often have prolonged courses of intravenous antibiotics. Selective decontamination could have a role to play in elective gastrointestinal surgical procedures and ongoing clinical trials are underway to examine this further. Whilst an exciting field, additionalmulti-centre work for specific indications such as elective GI surgery and any effect on long-term antibiotic resistance needs to be determined.

\section{References}

1. Thaiss CA, Zmora N, Levy M, et al. The microbiome and innate immunity. Nature. 2016;535(7610):65-74.

2. Price R, MacLennan G, Glen J, et al. Selective digestive or oropharyngeal decontamination and topical oropharyngeal chlorhexidine for prevention of death in general intensive care: systematic review and network meta-analysis. BMJ. 2014;348:g2197.

3. Roos D, Dijksman LM, Oudemans-van Straaten HM, et al. Randomized clinical trial of perioperative selective decontamination of the digestive tract versus placebo in elective gastrointestinal surgery. $\mathrm{Br}$ J Surg. 2011;98(10):1365-1372.

4. Diepenhorst GM, van Ruler O, Besselink MG, et al. Influence of prophylactic probiotics and selective decontamination on bacterial translocation in patients undergoing pancreatic surgery: a randomized controlled trial. Shock. 2011;35(1):9-16.

5. Lingnau W, Berger J, Javorsky F, et al. Changing bacterial ecology during a five-year period of selective intestinal decontamination. $J$ Hosp Infect. 1998;39(3):195-206. 
6. Sleijfer DT, Mulder NH, de Vries-Hospers HG, et al. Infection prevention in granulocytopenic patients by selective decontamination of the digestive tract. Eur J Cancer. 1980;16(6):859-869.

7. Mulder JG, Wiersma WE, Welling GW, et al. Low dose oral tobramycin treatment forselective decontamination of the digestive tract: a study in human volunteers. J Antimicrob Chemother. 1984;13(5):495-504.

8. van der Waaj D, Gaus W, Krieger D, et al. Bacteriological data on a prospective multicenter study of the effect of two different regimens forselective decontamination in patients with acute leukaemia. Infection. 1986;14(6):268-274.

9. Ledingham IM, Alcock SR, Eastaway AT, et al. Triple regimen of selective decontamination of the digestive tract, systemic cefotaxime, and microbiological surveillance for prevention of acquired infection in intensive care. Lancet. 1988;1(8589):785-790.

10. Ulrich C, Harinck-de Weerd JE, Bkker NC, et al. Selective decontamination of the digestive tract with norfloxacin in the prevention of ICU-acquired infections: a prospective randomized study. Intensive Care Med. 1989;15(7):424-431.

11. Cockerill FR 3rd, Muller SR, Anhalt JP, et al. Prevention of infection in critically ill patients by selective decontamination of the digestive tract. Ann Intern Med. 1992;117(7):545-553.

12. Gianotti L, Munda R, Gennari R, et al. Effect of different regimens of gut decontamination on bacterial translocation and mortality in experimental acute pancreatitis. Eur J Surg. 1995;161(2):85-92.

13. deJonge E, Schultz MJ, Spanjaard L, et al. Effects of selective decontamination of digestive tract on mortality and acquisition of resistant bacteria in intensive care: a randomised controlled trial. Lancet. 2003;362(9389):1011-1016.

14. Silvestri L, van Saene HK, Milanese M, et al. Prevention of MRSA pneumonia by oral vancomycin decontamination: a randomised trial. EurRespir J. 2004;23(6):921-926.

15. Oostdijk EA, de Smet AM, Kesecioglu J, et al. The role of intestinal colonization with gram-negative bacteria as a source for intensive care unit-acquired bacteremia. Crit Care Med. 2011;39(5):961-966.

16. De Smet AM, Kluytmans JA, Blok HE, et al. Selective digestive tract decontamination and selective oropharyngeal decontamination and antibiotic resistance in patients in intensive-care units: an open-label, clustered group-randomised, crossover study. Lancet Infect Dis. 2011;11(5):372-380.

17. Hammond JM, Potgieter PD. Long-term effects of selective decontamination on antimicrobial resistance. Crit Care Med. 1995;23(4):637-645.

18. Noteboom Y, Ong DS, Oostdijk EA, et al. Antibiotic-Induced WithinHost Resistance Development of Gram-Negative Bacteria in Patients Receiving Selective Decontamination or Standard Care. Crit Care Med. 2015;43(12):2582-2588.
19. Oostdijk EA, Kesecioglu J, Schultz MJ, etal. Effects of decontamination of the oropharynx and intestinal tract on antibiotic resistance in ICUs: a randomized clinical trial. JAMA. 2014;312(14):1429-1437.

20. Vrieze A, Out C, Fuentes S, et al. Impact of oral vancomycin on gut microbiota, bile acid metabolism, and insulin sensitivity. J Hepatol. 2014;60(4):824-831.

21. Sanchez-Garcia M, Cambronero Galache JA, Lopez Diaz J, et al. Effectiveness and cost of selective decontamination of the digestive tract in critically ill intubated patients. A randomized, double-blind, placebo-controlled, multicenter trial. Am J Resp Crit Care Med. 1998;158(3):908-916.

22. Melsen WG, de Smet AM, Kluytmans JA, et al Selective decontamination of the oral and digestive tract in surgical versus non-surgical patients in intensive care in a cluster-randomized trial. Br J Surg. 2012;99(2):232-237.

23. Dijksman LM, Roos D, Gerhards MF, et al. Cost-effectiveness of perioperative selective decontamination of the digestive tract versus placebo in elective gastrointestinal surgery. Dig Surg. 2012;29(5):384 390

24. Abis GS, Oosterling SJ, Stockmann HB, et al. Perioperative selective decontamination of the digestive tract and standard antibiotic prophylaxis versus standard antibiotic prophylaxis alone in elective colorectal cancer patients. Danish Med J. 2014;61(4):A4695.

25. Ruza F, Alvarado F, Herruzo R, et al. Prevention of nosocomial infection in a pediatric intensive care unit (PICU) through the use of selectivedigestive decontamination. Eur $J$ Epidemiol. 1998;14(7):719-727.

26. Zobel G, Kuttnig M, Grubbauer HM, et al. Reduction of colonization and infection rate during pediatric intensive care by selective decontamination of thedigestive tract. Crit Care Med. 1991;19(10):1242-1246.

27. Chaari A, Zribi E, Dammak H, et al. Does selective digestive decontamination prevent ventilator-associated pneumonia in trauma patients? Am J Therapy. 2014;21(6):470-476.

28. Stoutenbeek CP, van Saene HK, Little RA, et al. The effect of selective decontamination of the digestive tract on mortality in multiple trauma patients: a multicenter randomized controlled trial. Intensive Care Med. 2007;33(2):261-270.

29. de la Cal MA, Cerda E, Garcia-Hierro P, et al. Survival benefit in critically ill burned patients receiving selective decontamination of the digestive tract: a randomized, placebo-controlled, double-blind trial. Ann Surg. 2005;241(3):424-430.

30. Gosney M, Martin MV, Weight AE. The role of selective decontamination of the digestive tract in acute stroke. Age Ageing. 2006;35(1):42-47. 